\title{
DINAMICA TERRITORIAL Y SOCIODEMOGRÁFICA DE LA MONTAÑA GALLEGA
}

\author{
José A. ALDREY VÁZQUEZ - Rubén C. LOIS GONZÁLEZ \\ Departamento de Xeografía y Geología, Universidad de Santiago \\ José $\mathrm{M}^{\mathrm{a}}$. SAN ROMÁN RODRÍGUEZ \\ Departamento de Geografía y Geología. Universidad de León
}

Recibido: $18 / 04 / 2010$

Aceptado: 27/04/2010

RESUMEN: En este artículo se realiza una aproximación a la dinámica territorial, demográfica y socioeconómica que viven los espacios de montaña en Galicia. Para ello se comienza definiendo lo que se entiende por montaña en esta Comunidad Autónoma a partir de la literatura geográfica existente sobre el tema. A partir de ahí se realiza un análisis del severo declive demográfico que se ha dado en estos territorios, que en la actualidad presentan una despoblación de magnitudes muy notables, con abandono de numerosos núcleos y con un colectivo humano profundamente envejecido. La dinámica económica de los espacios serranos gallegos es también abordada. Son áreas con grandes potencialidades inherentes, pero con un escaso desarrollo de las mismas, a excepción de actividades puntuales, como consecuencia de las dificultades para atraer inversores y de la fuerte atonía existente, muchas veces motivada por las propias características de una población mayor con escasa capacidad emprendedora y de innovación. A continuación se realiza una aproximación a los escasos ejemplos de iniciativas empresariales exitosas, que servirán para transmitir las posibilidades de futuro que existen para estos territorios en declive. Finalmente, se realiza una reflexión a modo de conclusión en la que se recapitula sobre lo apuntado en el artículo, que supone una puesta al día sobre la montaña en el desequilibrado territorio gallego. PALABRAS CLAVE: Montaña, Galicia, declive, despoblación, economía.

\section{TERRITORIAL AND DEMOGRAPHIC DYNAMICS OF THE GALICIANMOUNTAIN}

ABSTRACT: The present paper deals with an approximation to the territorial, demographic and socio-economic dynamic experienced by Galician mountain spaces. First of all, it is defined what mountain means in this Autonomous Community by making reference to the existing geographical literature. Starting from this, it is realized an analysis of the sharp demographic decline recorded in these territories, which nowadays present depopulation of outstanding magnitudes, with abandonment of numerous nucleuses and strongly aged human collectivity. The economic dynamic of Galician highlands spaces is taken into consideration, too. These areas have big inherent potentialities, but scarce development, excepted for punctual activities carried on in order to capture investors' attention. Furthermore, these areas present strong atony, which is mainly due to the characteristic of the same aged population with scarce entrepreneur and innovation ability. Then, it follows an approximation to the few examples of the successful business initiatives that were useful to show existing future possibilities for these declining 
territories. Finally, reflections conclude and sum up what stated in the article, which presupposes an updating about mountain and the unbalanced Galician territory.

KEY WORDS: Mountain, Galicia, Decline, Depopulation, Economy.

\section{DYNAMIQUE TERRITORIALE ET SOCIO-DÉMOGRAPHIQUE DE LA MONTAGNE GALICIENNE}

$\boldsymbol{R} \boldsymbol{E} \boldsymbol{S U} \boldsymbol{M} \boldsymbol{E}:$ Cet article est une réflexion sur les dynamiques territoriales, démographiques et socio-économiques aux espaces de montagne de la Galice. On définit d'abord ce que l'on veut dire quand on fait réference à la montagne dans cette Communauté Autonôme. Cette tâche de délimitation conceptuelle est abordée après une lecture de plusieurs ouvrages géographiques sur la matière. À suivre, on introduit une analyse sur le profond déclin démographique subi dans ces territoires, en plein processus de depeuplement à cause de l'abandon de nombreux villages. En effet, le vieillissement de la population s'avère comme une de leurs plus fortes problématiques. En ce que concerne la dynamique économique, on remarque qu'il s'agit d'aires où on trouve de grands potentiels mais ceux-ci ne sont pas suffissament développés, hormis certaines activités ponctuelles. Cette situation est la conséquence des difficultés pour attirer des investissements et de l'apathie générale d'une population agée et peu ouverte à l'innovation. En suite, on réalise une approche sur les peu nombreux examples d'initiatives entrepreuneuriales qui ont eu du succès sur le terrain, pour montrer les possibilités d'avenir existantes dans ces territoires en déclin. Finalement, on conclut avec une synthèse des arguments exposés tout au long de l'article. Celuici est une mise-au-jour sur le rôle des montagnes au sein d'un territoire déséquilibré comme le galicien.

MOTS-CLÉS: montagne, Galice, déclin, depeuplement, économie.

\section{DINAMICA TERRITORIAL E SOCIO-DEMOGRÁFICA DA MONTANHA E GALEGA}

RESUMO: Neste artigo se faz uma aproximação à dinâmica territorial, demográfica e socioeconômica que vive os espaços de montanha na Galíza. Deste modo começa a ser definido o que se entende por montanha nesta Comunidade Autônoma partindo da literatura geográfica existente sobre o tema. A partir de então se realiza uma análise do severo declínio demográfico que se produziu neste território, apresentando na actualidade um processo de vazio populacional de magnitudes significativas, notando-se o abandono de numerosos núcleos de assentamentos e com o coletivo humano bastante envelhecido. A dinâmica econômica dos espaços serranos galegos é também abordada; são áreas com grandes potencialidades próprias por muitas vezes motivadas pelas características de uma população envelhecida com pouca capacidade empreendedora e de inovação. A continuação se realiza uma aproximação aos escassos exemplos de iniciativas empresariais que tenham obtido êxito, servindo assim para transmitir as possibilidades de futuro que existe para estes territórios em declive. Finalmente se realiza uma reflexão como conclusão onde se recapitula sobre o que foi descrito no artigo que se supõe uma atualização sobre a montanha no desequilibrado território galego.

PALAVRAS-CHAVES: Montanha, Galíza, declínio, vazio, economia.

Desde la Edad Media y el primer gran período de peregrinaciones jacobeas, Galicia ha sido definido como un país de montes, colinas y continuos cambios topográficos. Esta imagen de la Comunidad Autónoma que ocupa el extremo Noroeste ibérico fue reiterada por numerosos viajeros que la descubrían, por las primeras descripciones y análisis geográficos de los siglos XIX y XX, por los 
pintores galeguistas de los años 1920 y 1930, y se ha mantenido sin excesivas variaciones hasta la actualidad. De hecho, en las imágenes promocionales del territorio se suele acudir a paisajes verdes intensos, de montes o de grandes moles montañosas, para sintetizar la percepción idealizada que se mantiene del mismo. La afirmación montuosa de Galicia es lógica, al menos por dos razones. En primer lugar, como contraste con la planitud dominante en la Meseta castellana, de modo particular en la Cuenca del Duero. En segundo término, como asociación subconsciente con otras regiones y países atlánticos europeos (Cantabria, Bretaña, Escocia, Cornualles, etc.), donde la influencia oceánica (céltica si recurrimos a referentes culturales más o menos míticos), se suele traducir en perfiles ondulados del horizonte, en pastos de altura y con marcadas pendientes, y en abundancia de acantilados. No obstante, a pesar de esta arraigada imagen no toda Galicia debe considerarse ni mucho menos terreno montañoso. Por una parte, el amplio espacio litoral del país excluye este calificativo, aún cuando las áreas próximas a las rías muestren una relativa variación altitudinal entre valles, llanuras elevadas y algunos montes. Por otra, porque en las cuatro provincias existen amplios espacios más o menos planos, escasamente utilizados en las imágenes de comercialización turística hacia el exterior, pero no por ello poco importantes en la configuración del territorio (la Terra Chá lucense, A Limia ourensana, la comarca del Xallas entre Santiago y la Costa da Morte, etc.). Esta evidencia ha justificado que diversos estudios geográficos sobre el país hayan ensayado (con bastante éxito) delimitar la montaña en Galicia, caracterizarla como un espacio con serios problemas, y plantear estrategias de corrección del declive de unas áreas todavía consideradas periféricas, poco accesibles y sin una estrategia clara de desarrollo futuro. En la presente contribución trataremos de repetir este esquema, asumiendo que la montaña es tan solo una porción minoritaria de la Comunidad Autónoma, insistiendo en que se trata de los municipios con mayores índices de despoblación, envejecimiento y atonía económica, y planteando de cara al futuro algunos ejemplos (aislados) de desarrollo endógeno que se han acometido en estos sectores durante los últimos tiempos.

A partir del discurso que acabamos de enunciar, el presente artículo se organizará en cuatro epígrafes y una conclusión final. El apartado inicial se consagrará a definir la montaña, los territorios de montaña en Galicia, siguiendo para este fin dos publicaciones de referencia sobre el tema: el libro A Montaña Galega. O home e o medio que en 1993 se editó por parte de la Universidad de Santiago y la obra colectiva del grupo CIMA, sobre Las montañas españolas: conceptos y vocabulario (TORRES, LOIS y PÉREZ ALBERTI, 1993; CIMA, 2005). En ambas contribuciones participó el más veterano de los tres autores del presente artículo, por lo que su contenido junto a otro incluido en el número monográfico de ERIA sobre el tema, constituye la continuación de unas tesis formuladas hace casi veinte años (ERIA, 2008; LOIS, SAN ROMÁN y ALDREY, 2008). En un 
segundo epígrafe nos interesaremos por la magnitud del declive demográfico de la montaña en nuestro país. A comienzos del siglo XXI nos encontramos con un conjunto de territorios que sufren una aguda despoblación, el abandono de numerosas aldeas y casas, y que a día de hoy son ocupados por una población compuesta hegemónicamente por mayores; la montaña como expresión acrecentada de la decadencia del viejo mundo rural del Noroeste ibérico. En tercer lugar, se comprobará cómo la crisis demográfica se refleja en la dinámica económica, pero de una forma más matizada. Las áreas de montaña han conseguido animar ciertas ramas productivas adaptadas a la realidad actual, partiendo de sus potencialidades inherentes (abundancia y riqueza de recursos de calidad), y recurriendo en muchos casos a trabajadores y empresarios que viven fuera de las áreas más aisladas, pero que desarrollan en las mismas el grueso de su actividad. De hecho, algunos de los ejemplos exitosos (y aislados) de desarrollo en espacios de montaña serán utilizados en el cuarto apartado, para insistir en las posibilidades de futuro que se abren para estas comarcas (como ya sucede en algunos ejemplos españoles y europeos). Por último, las conclusiones finales constituirán un ejercicio de reflexión y puesta al día sobre la montaña en una Galicia territorialmente muy desequilibrada entre los espacios urbanos y costeros, y los extendidos por las áreas rurales del interior.

\section{CONCEPTO Y CARACTERIZACIÓN DE LA MONTAÑA EN GALICIA}

Para la Geografía, la definición de montaña ha sido un tema clásico y recurrente, por lo que existen una serie de acuerdos sobre sus caracteres. Así, se considera que la montaña no es sólo una realidad física, una forma concreta de relieve, que puede presentarse aislada o formando un conjunto, una alineación, cordal, cordillera o sierra (MARTíNEZ DE PISÓN, 1981; MUÑOZ y SANZ, 1995). Resulta evidente que lo montañoso engloba todas estas acepciones, pero al mismo tiempo montaña es una imagen, que ha sido recreada por numerosos artistas y en múltiples documentales (LÓPEZ SILVESTRE y SOBRINO, 2006), y una acepción que se contrapone a llanura, a los espacios donde la planitud de las formas domina el paisaje (GARCÍA FERNÁNDEZ, 1985). En Galicia, y así se apuntaba al comienzo, en el estudio de $\mathrm{M}^{\mathrm{a}}$.P. de Torres, R.C. Lois y A. Pérez Alberti se evocaba la consideración popular de montaña como todo lo que no es litoral o no es un área resguardada (TORRES, LOIS y PÉREZ ALBERTI, 1993). Un significado de montaña que también presenta connotaciones negativas: lo montañés, os da montaña, suelen verse como más rudos, atrasados, siguiendo las antiguas lógicas de contraposición de las sociedades civilizadas con las otras, las denominadas bárbaras. En todo caso, montaña y montañoso hacen referencia a lugares elevados, en resalte sobre los territorios de su alrededor. También a las formas de vida que se han desarrollado en estos espacios, caracterizadas 
casi siempre por una adaptación dificultosa a las condiciones impuestas por un medio natural duro. La montaña es el mundo de las áreas con superior altitud, aunque no exista un límite asumido con carácter general para separar lo montañoso de lo que no lo es. De forma complementaria, montaña es un área expuesta a los vientos dominantes y asociada a una intensa pluviosidad, y un espacio definido por fuertes pendientes, que dificultan el asentamiento humano y la materialización de determinadas actividades económicas (CIMA, 2002). Aunque el término montaña permite individualizar a un conjunto amplio de espacios con rasgos bien singularizados, sus límites no son tajantes tanto respecto a otras áreas como entre los diversos tipos de sectores montañosos. Por lo tanto, es frecuente que montaña se llegue a cualificar de muy diferentes formas. Existe la alta montaña y la montaña media; la primera, como montaña con todos sus atributos, la segunda como una expresión que trata de matizar las trazas de la altitud, exposición y pendiente. También podemos referirnos a montañas atlánticas o mediterráneas, orientales o septentrionales, montañas húmedas o secas, entre diversas posibilidades (CIMA, 2005), algunas de las cuales utilizaremos (de forma explícita o implícita) en nuestro trabajo.

Sin lugar a dudas, en Europa, España y Galicia la acepción montaña se ha convertido así mismo en una categoría de ordenación del territorio (Grupo ADUAR, 2000). De hecho, desde 1975 en que se aprobó la Directiva de la CEE sobre agricultura de montaña y zonas desfavorecidas, lo montañoso ha sido individualizado en distintas ocasiones para intervenir en espacios aquejados de limitaciones físicas para la práctica de la actividad agraria, condicionados por su aislamiento, definidos por encuadrarse entre los sectores más característicos de las bajas densidades, y de forma habitual considerados como atrasados en términos de desarrollo. Respecto a este último significado, cabe precisar que no todas las áreas de montaña presentan problemas económicos derivados de sus rasgos constitutivos, ya que en algunas de ellas la consolidación del turismo deportivo y de montaña se ha convertido en un factor muy importante de generación de riqueza. No obstante, aún teniendo en cuenta esta situación representativa de muchos espacios alpinos, pirenaicos o de la Cordillera Cantábrica, la montaña como territorio singular a ordenar siempre se ha interpretado en términos de dificultades, de conjunto de sectores poco favorecidos por su altitud, pendientes y exposición a agentes meteorológicos que pueden ser dañinos. Así, si la Directiva de 1975 abrió el camino a la delimitación de la montaña con una finalidad de ordenación, la ley española de agricultura de montaña de 1982 procuró adaptar la legislación estatal a un marco comunitario en el que era inminente nuestra integración, y el Reglamento del Consejo Europeo de 1999 sobre ayudas al desarrollo rural a cargo del FEOGA, ha ratificado esta interpretación de los territorios de montaña como necesitados de ayuda (CIMA, 2005). En estos casos, y también en las iniciativas tomadas con motivo de la celebración del 
Año Internacional de las Montañas de 2002, lo montañoso es considerado como una realidad que impone severas dificultades, tanto por unas variantes climáticas que acortan el período vegetativo, como por la presencia de fuertes desniveles que limitan el uso de los terrenos o por las dificultades de acceso a numerosos lugares (CEE, 1975). Esta constatación ha justificado que existiese una voluntad de delimitar las comarcas o municipios de montaña a partir de cierta altitud (por encima de 800 o 1.000 metros), con presencia de pendientes de entidad (más del $20 \%$ a nivel local) y con rendimientos por superficie mucho menores que los espacios agrícolas que podían ser considerados normales o estándares.

Las zonas de montaña se han definido siguiendo, con uno u otro indicador numérico, estas variables. De esta forma, en España desde comienzos de los 1980 existe una clasificación de áreas de montaña, construida a partir de la escala municipal y aceptada por la literatura geográfica sobre el tema (TORRES, LOIS y PÉREZ ALBERTI, 1993; CIMA, 2005), aún cuando no haya sido muy operativa en términos prácticos de desarrollo rural. Como es sobradamente conocido, el empleo de criterios de homogeneidad territorial (áreas de montaña, marginales, insulares, zonas árticas, etc.) en desarrollo local y rural en el ámbito de la UE es limitado. En concreto, desde hace decenios se ha preferido respaldar a agrupaciones supramunicipales de carácter voluntarista (impulsadas por Grupos de Acción Local o por ayuntamientos) para materializar los grandes programas de intervención sobre unos espacios agrarios que iban quedando descolgados de los grandes ejes de crecimiento conformados en nuestro continente (o en España). Así, las áreas LEADER, PRODER u objeto de iniciativas comunitarias como INTERREG apenas han recurrido a las delimitaciones de la montaña que, sin embargo, si se han mantenido en el imaginario público y en la bibliografía especializada sobre el rural con mayores dificultades de inserción en las nuevas lógicas de organización del espacio (CABERO, MAJORAL y RODRÍGUEZ MARTíNEZ, 1992).

Esta serie de aclaraciones a nivel general fueron realizadas, de un modo necesariamente sintético, para Galicia a comienzos de los 1990 (TORRES, LOIS y PÉREZ AlBERTI, 1993). De hecho, aunque se asumía que la ordenación del territorio rural seguía otras dinámicas de agrupación supramunicipal diferentes, esto no fue obstáculo para que se plantease analizar y diagnosticar la montaña gallega como una realidad bien singularizada, partiendo para ello de la delimitación de Zonas de Agricultura de Montaña que el gobierno español reguló, de forma sucesiva, en los años 1980. Como resultado de este proceso, en la Comunidad Autónoma cerca de 120 municipios, el 44,2\% de la superficie total, se catalogaban como de montaña. Una delimitación considerada excesiva en el estudio de referencia, que optó por excluir de la clasificación territorios aplanados del Sur lucense (Pantón, Sober, Carballedo, etc.), donde la única característica 
montañosa observable eran las fuertes pendientes que se extienden en las riberas del Miño y del Sil, la comarca de Valdeorras (que como indica su nombre constituye un espacio de valle entre montañas), la cuenca media del Ulla (terras de Arzúa-Melide), la depresión de Verín y algunos municipios aislados (Xinzo de Limia, Arbo, Padrenda, etc.), donde el dominio de superficies llanas resulta evidente (TORRES, LOIS y PÉREZ ALBERTI, 1993). La consecuencia de este proceso de filtrado ha sido que en Galicia la mayoría de los estudios sobre la cuestión han calificado de montaña un total de 84 términos municipales, distribuidos por las cuatro provincias (FIGURA 1) (TORRES, LOIS y PÉREZ ALBERTI, 1993). Se trata de los territorios que se extienden por las denominadas Serras Orientais y Sudorientais, que sirven de separación natural con la Comunidad Autónoma de Castilla y León, de una serie de espacios próximos de alta y media montaña que ocupan la mitad Este y Sur de la provincia de Ourense (en Manzaneda, la Serra do Xurés, las tierras altas de Verín-A Gudiña, etc.), y de los espacios articulados por la Dorsal gallega, un conjunto de relieves entre los 800 y los 1.200 $\mathrm{m}$. de altitud, que se extienden en dirección meridiana por el límite de las provincias de A Coruña y Pontevedra con Lugo y Ourense.

El mapa municipal de la montaña gallega individualiza a los sectores más elevados, con mayores pendientes y problemas de aislamiento de la Comunidad Autónoma. A este respecto, es conocido que todo el espacio que sirve para separar Galicia respecto a los territorios vecinos (el Principado de Asturias, Castilla y León, y las áreas portuguesas del Alto Douro y Tras-OsMontes) concentra los sistemas montañosos más importantes del territorio. Por una parte, los antiguamente denominados (en numerosas Geografía de España) montes (o macizo) gallego-leonés, que en Galicia se conocen de forma mayo-

Figura 1. Ubicación de los espacios de montaña en Galicia

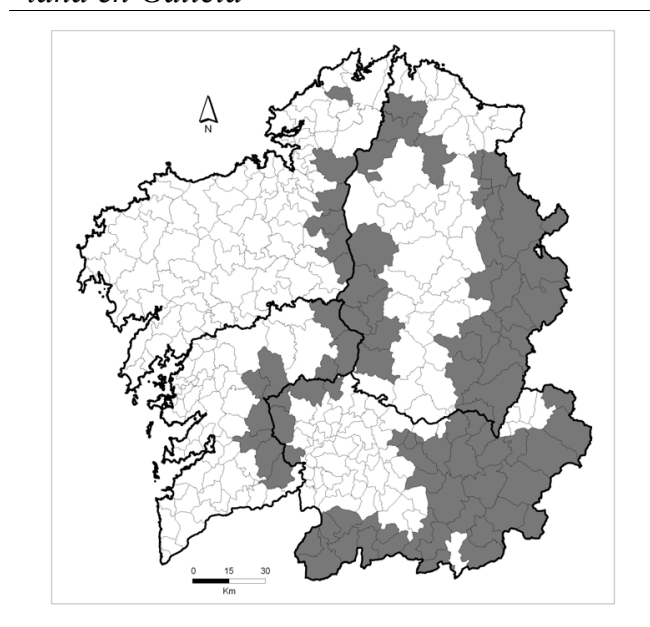

FUENTE: Basado en TORRES, LOIS y PÉREZ AlberTi, 1993 ritaria como las Serras Orientais (desde A Fonsagrada hasta el Caurel, pasando por el Cebreiro y Ancares), Sudorientais (Manzaneda, O Eixo, Segundeira, Trevinca, San Mamede, etc.) y Meridionais (Xurés, Leboreiro, Larouco, etc.) (Torres, Lois y Pérez Alberti, 1993). Estos espacios conformarían lo que en la Comunidad Autónoma se ha denominado alta montaña, áreas con una altitud comprendida entre los 1.200 y los 2.000 metros, escasos niveles de ocupación hu- 
mana (densidades inferiores a $10 \mathrm{hab} / \mathrm{km}^{2}$ ) y severos problemas de accesibilidad. El resto de la montaña se corresponde con municipios situados en las estribaciones de la Dorsal (desde A Capelada hasta la Serra do Suído pasando por el Faro, Cova da Serpe, el Farelo, O Careón, etc.) y las Serras Septentrionais (Xistral, Montes do Buio, etc.), que sirven de límite a la Terra Chá con la Mariña de Lugo. Se trata de sectores de la montaña media, de 800 a $1.200 \mathrm{~m}$. de altitud, con desniveles topográficos menos acusados y que en muchos aspectos se asemejan a otros lugares de la Galicia rural e interior.

En este recorrido por las áreas de montaña, sólo nos resta comentar que las mismas engloban a cerca de 40 términos municipales ourensanos, que se extienden por toda la mitad oriental de la provincia (comarcas de O Bolo, Manzaneda y Trives, los confines de Valdeorras, etc.), prácticamente todo su borde meridional (en Verín, A Gudiña y A Baixa Limia), y algunos sectores aislados del Noroeste (periferias de la Terra do Carballiño y O Ribeiro). En Lugo, el numero de territorios montañosos es algo inferior (no alcanzan los 30), divididos entre los correspondientes a las Serras Orientais (desde la comarca de Quiroga-O Caurel hasta A Fonsagrada-Burón) y los menos agrestes de la Dorsal y las Serras Septentrionais (Chantada, A Ulloa y límites noroccidentales de A Terra Chá). En Pontevedra se han considerado montañosos 9 términos municipales, de su extremo oriental (desde O Covelo en el Sur hasta Agolada en el Norte), lo mismo que las 6 entidades pertenecientes a Coruña (Toques, Sobrado dos Monxes, Curtis, Aranga, Monfero y Cerdido). En general, la montaña explica las dinámicas territoriales seguidas por una parte significativa de Lugo y Ourense, mientras que en Pontevedra y A Coruña sólo representa a sectores muy periféricos y rurales de los rebordes provinciales.

\section{ALGUNOS RASGOS BÁSICOS DE LA DINÁMICA DEMOGRÁFICA DE LAS ÁREAS DE MONTAÑA}

Los 84 municipios definidos como de montaña en Galicia expresan perfectamente los caracteres de regresividad poblacional y envejecimiento acusado que se suelen adscribir a las áreas del rural menos dinámico. De hecho, la población total de este conjunto de territorios sólo alcanza los 178.684 hab. en 2009, un mínimo 6,4\% del total de la Comunidad Autónoma. Quizás el dato más significativo a este respecto sea que el efectivo humano de estos territorios disminuyó un 55,3\% desde 1960, mientras que en Galicia el total de población se mantenía básicamente estancada (un incremento del 2,3\% de 1960 a 2009). En concreto, se debe anotar que las ganancias demográficas correspondientes a las provincias de A Coruña y Pontevedra son compensadas por pérdidas severas en Lugo ($27,8 \%$ en el mismo período) y, en particular, Ourense (-31,2\%). En cualquier caso unos valores todavía alejados del dramatismo de los municipios de monta- 
ña, donde se puede hablar de una catástrofe sociodemográfica por cuanto las pequeñas comunidades rurales de antaño (las parroquias), cerradas sobre si mismas y representativas de un viejo complejo agrario-rural (Torres y Lois, 2009) se han ido diluyendo, entre otros factores, por la magnitud del retroceso poblacional. El éxodo rural iniciado a mediados del siglo XX, una emigración dirigida hacia regiones prósperas del estado (en particular Cataluña) y de Europa (Suiza, Francia, etc.), vació las áreas de montaña gallegas. En este proceso, fue especialmente dura la salida masiva de adultos-jóvenes que se incorporaban al mercado de trabajo y que dejaban a los territorios de montaña como un espacio, ya en los 1960-70, mucho más envejecido que su entorno y donde la falta de iniciativas económicas se correlacionaba con la escasez de individuos en edad de acometer procesos de modernización productiva (casi siempre de base rural) (LOIS y RODRÍGUEZ, 2001).

Desde finales de los 1970 los flujos migratorios hacia el exterior no son tan visibles, aunque se han mantenido de una forma más sutil. De la montaña gallega marchan más mujeres, en dinámicas individuales con dificultad para ser detectadas, y que se dirigen hacia ciudades próximas (Vigo, A Coruña, Ourense o Lugo, como grandes receptores) o hacia grandes espacios urbanos percibidos como muy atractivos (Madrid, Barcelona, etc.). Sin embargo, estos flujos migratorios han perdido protagonismo en la explicación mayoritaria del declive demográfico reciente de los 84 municipios estudiados (un retroceso del 17,8\% de 1998 a 2009, cuando se ha establecido el Padrón de Habitantes continuo), que obedece en buena medida al intenso proceso de envejecimiento de estos territorios. De hecho, si en Galicia ya un 21,7\% de la población supera los 65 años, lo que constituye una cifra estimable, que expresa las dificultades demográficas del conjunto de la Comunidad Autónoma, en la montaña este porcentaje se eleva hasta un alarmante $37,8 \%$. Un dato que resalta todavía más si lo comparamos con el escaso 9,3\% de menores de 20 años. Atendiendo a la contundencia de estos registros, se puede manifestar que los espacios de montaña se individualizan en la actualidad como lugares de viejos, donde las estructuras demográficas y familiares mínimamente equilibradas han sido destruidas por un proceso iniciado a mediados del siglo XX, que generó primero una emigración masiva, luego una pérdida selectiva de habitantes y que hoy dibuja un panorama sin apenas futuro, porque aunque las coyunturas de crisis como la actual pudiesen animar la instalación de familias jóvenes procedentes de ámbitos urbanos en algunos de los territorios estudiados, este fenómeno de reasentamiento poblacional no cubriría ni en parte el crecimiento natural muy negativo de unas áreas aquejadas por un envejecimiento general del colectivo humano que las habita (ALDREY, 2009). 
Figura 2. Distribución municipal de los menores de 20 años (a) y de los mayores de 65 años (B) en los espacios de montaña en Galicia en 2009.

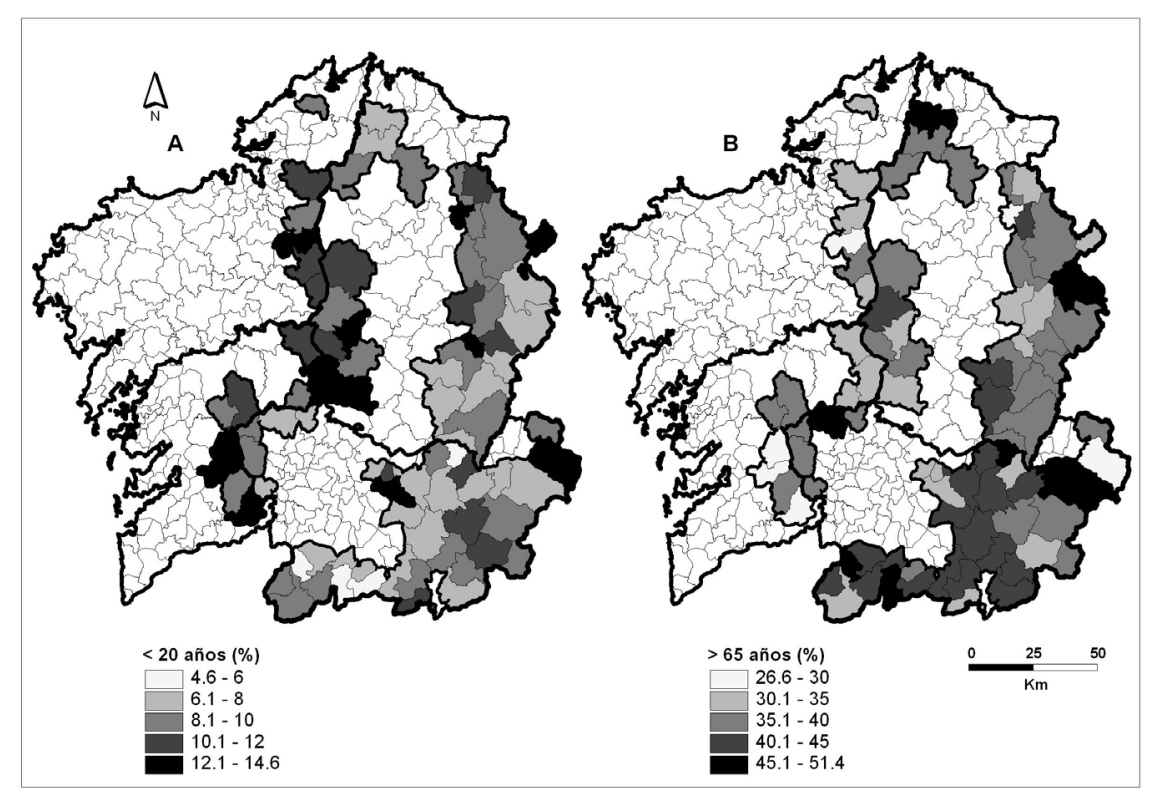

FUENTE: Padrón de habitantes, 2009.

Un análisis somero de la distribución municipal de los porcentajes de mayores de 65 años en la montaña (FIGURA 2A), nos muestra que apenas el territorio minero de Carballeda de Valdeorras, que se ha beneficiado de la inmigración de portugueses para trabajar en la explotación de la pizarra (San Román, 2000 y 2005), se individualiza por debajo de un $30 \%$ de población de avanzada edad. Por el contrario, las cifras relativas de mayor envejecimiento sitúan a algunos términos con una proporción de personas que han cumplido los 65 superior al $45 \%$, un valor que anuncia una inminente desaparición de comunidades rurales y aldeanas en plenos Ancares (municipio de Navia de Suarna), Pena Trevinca (A Veiga), Manzaneda (San Xoán de Río), las Serras Meridionais (Calvos de Randín y Lobeira), las estribaciones ourensanas de la Dorsal (O Irixo) y lucenses de las Serras Septentrionais (Ourol). En todos estos casos estamos ante municipios muy rurales, sin apenas ninguna localidad de importancia que ejerza auténticamente de cabecera. Un conjunto de áreas tanto de la alta como de la media montañas, que manifiestan el hundimiento de la sociedad campesina tradicional que no ha sabido adaptarse a los procesos de modernización económica desarrollados desde los años 1960. Tanto en Ourol como en A Veiga, Navia de Suarna o Calvos de Randín, por citar algunos ejemplos, existen numerosos recursos territoriales dispuestos a ser explotados (magníficos paisajes que favore- 
cerían el impulso del turismo de la naturaleza, importantes extensiones de monte comunal aptos para acoger grandes rebaños de vacuno y ovino, bosques capaces de favorecer una explotación forestal sostenible, etc.), pero falta vocación de emprendimiento en colectividades viejas, derrotadas y sin apenas voluntad de innovación. Nos encontramos ante un ejemplo extremo de los severos problemas territoriales que aquejan a una Galicia que concentra su crecimiento poblacional y dinamismo económico en una estrecha franja litoral próxima al Atlántico, en tanto que el resto de los espacios siguen sufriendo intensas pérdidas poblacionales y problemas de estancamiento de sus principales sectores productivos. En la montaña, además, se plantea el problema formulado por diversos autores y asumido por la Federación Galega de Municipios y Provincias (FEGAMP), en relación con la falta de utilidad de muchas entidades locales que se están quedando vacías y donde la prestación de servicios en buenas condiciones al conjunto del efectivo humano (unos colectivos necesitados de mayor atención pública considerando su edad media) se vuelve cada vez menos factible (RODRÍGUEZ, 2004).

En la FIGURA 2B se presenta el mapa inverso al anterior, el que cartografía el porcentaje de personas que no han cumplido los 20 años en los municipios de montaña. De nuevo, los valores menos negativos vuelven a aparecer en Carballeda de Valdeorras, junto a una serie de territorios que envían a parte de sus trabajadores a ciudades bastante próximas (A Lama y Fornelos de Montes a Pontevedra, Maceda a Ourense), y otros centralizados por una cabecera dinámica (A Cañiza, Monterroso, Chantada, Meira) o donde se han generado posibilidades locales de empleo (en Curtis con cierta implantación industrial y la presencia de una cárcel, en Triacastela con el efecto del Camino de Santiago, en Rodeiro con la localización de algunas empresas, y en Negueira de Muñiz donde desde hace varios decenios funciona una comuna de base agraria). Por el contrario, la juventud demográfica es casi inexistente (del 4,6\% al 6\% de menores de 20 años), en varios municipios de la raya ourensana con Portugal (Lobeira, Calvos de Randín y Baltar) y de las estribaciones de Manzaneda (San Xoán de Río), que ya destacaban por sus elevados niveles de envejecimiento. El resto de la montaña gallega mantiene unos registros entre el $6 \%$ y el $12 \%$, que expresan claramente la falta de futuro poblacional de estas comunidades rurales aquejadas de una ausencia severa de vitalidad demográfica.

Para terminar con este diagnóstico sobre la situación de los colectivos humanos que habitan la montaña gallega, vamos a comentar dos datos muy reveladores de la dramática situación existente, que han sido obtenidos del Censo de Población y Viviendas de 2001 (y que es posible que sean mucho más negativos cuando, en 2011, se actualice esta fuente oficial de información). Se trata del porcentaje de hogares unipersonales ocupados por personas mayores de 65 
años, a nivel municipal (FIGURA 3A), y de unidades de residencia de uno o dos miembros, en los que uno de ellos supera el umbral de edad que indica la entrada en la vejez (FIGURA 3B).

Figura 3. Distribución municipal de los hogares unipersonales ocupados por mayores de 65 años (a) y de los hogares con dos miembros, con uno al menos por encima de 65 años (B) en los espacios de montaña en Galicia en 2001.
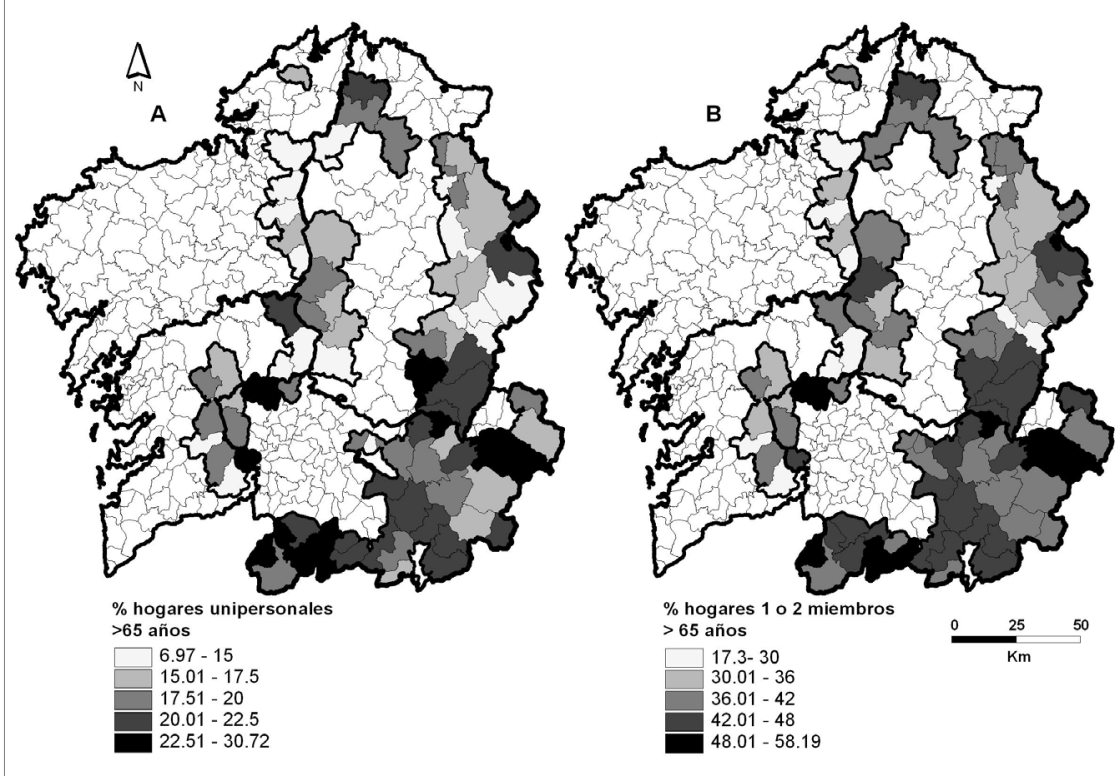

FUENTE: Censo de Población y Vivienda, 2001.

En el primer caso, sorprende la magnitud de situaciones donde una persona mayor vive sola, en un espacio rural normalmente afectado por problemas de accesibilidad. Esto sucede en el 18,6\% de los hogares de los 84 municipios de montaña, un valor que casi duplica el promedio de Galicia para esta eventualidad (el 10,5\%). Cuando aludimos a que las áreas más elevadas y de fuertes pendientes de la Comunidad Autónoma eran ocupadas por viejos, en aldeas o pequeños núcleos próximos a desaparecer, pensábamos en muchos ejemplos que se suceden en territorios como Lobeira, Entrimo, Calvos de Randín, A Porqueira, Muiños, Melón, O Irixo, San Xoán de Río y A Veiga, todos ellos en Ourense, donde el Censo de 2001 contabilizaba entre un $22,5 \%$ y un 30,7\% de hogares de solitarios con más de 65 años. Las cifras menos regresivas en función de este indicador vuelven a situarse en términos con cabeceras relativamente dinámicas (Chantada, Maceda, A Cañiza, Meira) o con ciertas posibilidades de desarrollo endógeno (los municipios xacobeos de Pedradita do Cebreiro y Tria- 
castela, con alguna implantación empresarial como Rodeiro, Dozón y Muras, etc.). En cualquier caso, y a pesar de constatarse excepciones, la mayoría de la montaña no sólo continuará despoblándose sino que verá desaparecer hogares a un ritmo acrecentado durante los próximos años, unos lugares de residencia que en muchos ejemplos son los últimos habitados de aldeas también condenadas a quedar vacías en su totalidad. Como ya apuntamos al inicio de este párrafo, junto al porcentaje de hogares de mayores solitarios, llama la atención por su magnitud el de unidades de habitación compuestas por una o dos personas, donde aparece un viejo: el 39,3\% del total en la montaña gallega frente a un $23,6 \%$ del conjunto de la Comunidad Autónoma. En este sentido, y para terminar, debemos ser conscientes de que ya en el 2001 aproximadamente la mitad de los hogares de A Veiga, San Xoán de Río, O Irixo, Lobeira, Calvos de Randín y Baltar, presentaban estas características alarmantes para el porvenir demográfico de los territorios.

\section{LAS ACTIVIDADES ECONÓMICAS DE LA MONTAÑA GALLEGA A PRINCIPIOS DEL SIGLO XXI}

De una forma semejante a lo ocurrido con la evolución demográfica, los años 1950 y 1960 supusieron el inicio de cambios muy profundos en la estructura productiva de las áreas de montaña gallegas. Por una parte, el viejo complejo agrario que se había mantenido durante siglos poco alterado, justificando un modo de vida campesino basado en una economía agraria diversificada (con el policultivo) y orientada hacia la subsistencia, comenzó a dar muestras de una crisis insalvable (BEIRAS, 1972; BOUHIER, 1979; COLINO y PÉREZ TOURIÑO, 1983). Al despoblarse los campos se hacía menos necesario producir para la propia casa, al mismo tiempo que las lógicas del sistema de mercado (obtener carnes baratas para hacer frente a las demandas urbanas en aumento) se abrían paso en todo el rural gallego. Desde estos decenios, el equilibrio entre agricultura, ganadería y rentabilización del monte en las explotaciones de montaña se rompe en dos direcciones: bien se registra una intensificación de la actividad pecuaria, a la que se subordinan todos los esfuerzos de la unidad productiva, bien se intentan obtener beneficios forestales de un monte extenso, cuyos rendimientos hasta ese momento no podían ser considerados importantes en términos monetarios. De hecho, lo que sucedió fue muy claro, las elevadas densidades rurales de población de estos territorios fueron desapareciendo al mismo tiempo que se generalizaba la emigración masiva hacia el exterior. Así, el objetivo del autoconsumo familiar perdía fuerza. Si a esto sumamos que la mejora de las comunicaciones y la accesibilidad permitió impulsar un creciente mercado de productos agrarios y forestales, nos encontraremos con un segundo factor de las fuertes transformaciones económicas que han tenido lugar. Como resultado de las mismas: el número de explotaciones agrarias tiende a reducirse (pero 
de forma moderada, en paralelo a la dinámica de desaparición de hogares); las unidades de producción que se mantienen optan por impulsar un rendimiento estrella que les permita competir en condiciones de apertura hacia el mercado (normalmente leche, carne o derivados forestales); se va generalizando el empleo al margen del sector primario, tanto en la industria de las proximidades, en cuadrillas de obreros de la construcción, o gracias al desarrollo de ciertos servicios (hostelería, turismo, puestos de trabajo públicos, etc.) y, por último, todos estos cambios se producen en un contexto de economías locales fuertemente subsidiadas (tanto por el montante de las pensiones que recibe el elevado número de mayores residentes en estos espacios, como por tratarse de municipios que se han beneficiado de la práctica totalidad de los planes de desarrollo rural impulsados por la UE y otras administraciones más próximas).

Esta descripción de los cambios operados en la economía de montaña en Galicia sería perfecta sino considerásemos que nos hayamos ante sectores menos accesibles, con inferior productividad de los suelos y con una actividad agraria más frágil que la de comarcas vecinas extendidas sobre espacios llanos o de va1le. La alta y la media montañas han sufrido enormes dificultades de adaptación a las nuevas lógicas productivas, se han convertido en periferia de un mundo rural que también ha perdido como resultado de los cambios económicos recientes, y donde la aguda crisis demográfica a la que nos hemos referido tampoco ha contribuido a mejorar el panorama. Como se analizará, en algunas áreas la moderna explotación ganadera y forestal de montaña se ha consolidado, ha florecido cierta actividad industrial y turística, pero en otros muchos sectores el hundimiento del viejo sistema de rentabilización campesino no se ha acompañado de la afirmación de nuevos modelos económicos rentables, y la atonía, la ausencia de una orientación agraria o empresarial clara hacia el mercado, condena a una buena parte de los municipios de la montaña gallega a vivir de las ayudas externas antes que de las dinámicas productivas autogeneradas.

Aunque no disponemos de unos datos oficiales más recientes, consultando el Censo Agrario de 1999 se pueden verificar las afirmaciones que acabamos de realizar. En primer término, en la montaña gallega se sigue contabilizando un elevado número de explotaciones agrarias (un total de 55.649, el 20,6\% de la Comunidad Autónoma), que ponen de manifiesto dos hechos: casi todos los hogares, al margen de cual sea su dedicación principal, siguen vinculándose a una unidad de producción campesina; el número de explotaciones tiende a decrecer, como en toda Galicia, al mismo tiempo que va disminuyendo el número de habitantes, si bien toda la sociedad de los municipios de montaña continua siendo por principio agricultora. Si pasamos ahora a los valores de distribución de la superficie agraria por grandes dedicaciones, también aportados por el Censo Agrario de 1999 (FIGURA 4), nos encontramos con que los cultivos (tierras labradas) poseen una importancia muy matizada, el 7,32\% frente al $12,68 \%$ de 
Galicia, donde ya gran parte de los sembrados (forrajes, praderas polifitas, etc.) están subordinados a la actividad pecuaria. La superficie de pastos permanentes es elevada (más del $21 \%$ tanto en la montaña como en el conjunto de la Comunidad Autónoma), y ha crecido en los últimos tiempos como expresión del desarrollo de la actividad pecuaria en todo el territorio. En cualquier caso, en la montaña las "otras tierras no forestales", es decir las amplias superficies de monte bajo, escasamente productivo en la actualidad, y que manifiesta los fenómenos de despoblación y abandono del campo, suponen el $48,23 \%$ del total en los 84 municipios considerados, mucho más que el promedio de las cuatro provincias (el 36,24\%). Por el contrario, el peso de las repoblaciones forestales es inferior (23,18\% frente al 29,64\%), si bien tiende a incrementarse del mismo modo que las extensiones consagradas a prados y pastos.

Figura 4. Distribución de la superficie agraria por grandes dedicaciones en Galicia y en sus espacios de montaña en 1999.

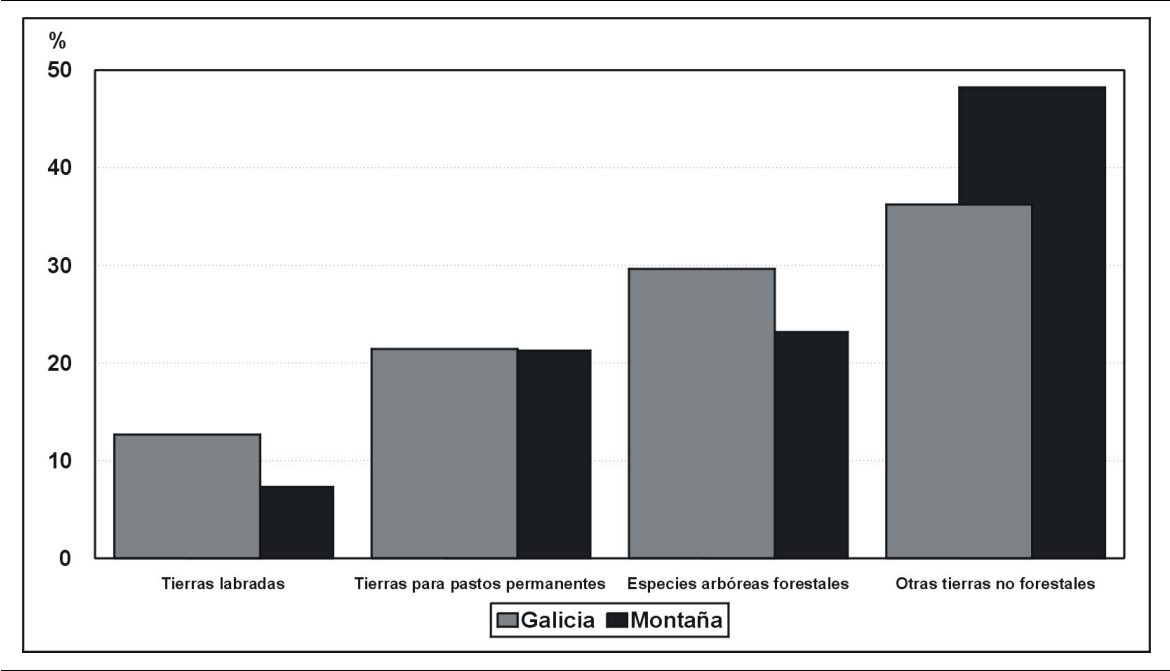

FUENTE: Censo Agrario, 1999.

En función de los valores comentados, la actividad agraria continúa siendo hegemónica en los espacios de montaña, y se orienta de forma mayoritaria a la explotación ganadera y a la rentabilización forestal. Por lo que se refiere a la cría pecuaria, y si recuperamos los registros del Censo Agrario de 1999, nos encontramos con que el bovino es la especie ampliamente hegemónica (220.318 Unidades Ganaderas), frente al porcino, confinado a un número limitado de granjas muy productivas (93.069 UG), y el ovino (11.789 UG), que apenas ha despegado a pesar de las enormes disponibilidades de espacios de monte comunal bien adaptados a sus necesidades de cría. Centrándonos en el bovino, aclarar que se distribuye entre un elevado número de explotaciones todavía supervi- 
vientes (11.552 en la montaña, según datos de 2008), la mayoría de las cuales se orienta hacia la producción de carne de calidad (una opción lógica y de futuro en espacios montañosos). De hecho, de un promedio de 23,11 bovinos por unidad agraria de montaña que se contabilizan en 2008 (un valor muy semejante al de Galicia, 19,24), sólo 5,85 vacas se clasifican de ordeño frente a 7,69 reservadas exclusivamente para cría. La actividad predominante de las explotaciones consiste en engordar terneros de razas autóctonas, alimentados parcialmente sobre los pastos de altura, como se demuestra con una media de 9,58 bovinos no reproductores por explotación. La vocación láctea ha disminuido mucho en épocas recientes (el número de vacas de ordeño ha descendido un $27,8 \%$ entre 2001 y 2008), como consecuencia de la inestabilidad del mercado y de las deficientes condiciones de accesibilidad para los camiones de recogida en numerosos lugares de montaña, mientras que la orientación cárnica se refuerza. En concreto, de 3,66 becerros de cría por explotación en 2001 se ha pasado a los comentados 7,69 en 2008, en un proceso que se acompaña de la recuperación de razas autóctonas o rústicas en las explotaciones, y de una menor dependencia de los sistemas de alimentación basados en el uso masivo de piensos adquiridos en el exterior.

Si la ganadería cárnica aparece como una de las escasas actividades con porvenir en una montaña aquejada de despoblación y de declive generalizado de la mayoría de las actividades productivas, algo similar se puede decir de la explotación forestal y de ciertas especializaciones agrícolas puntuales. El sector forestal se basa en la transformación maderera de las numerosas repoblaciones de pinos, que se han extendido por la Dorsal, las Serras Orientais y en menor medida las Sudorientais. En general, la montaña interior de Galicia sigue actuando como una reserva de árboles autóctonos (carballo, roble, castaño, abedul, etc.), con menor capacidad de producción, mientras que la especie foránea más agresiva del monte de la Comunidad, el eucalipto, apenas ha progresado en áreas de elevada altitud y fríos inviernos (MIRAMONTES, 2009). No obstante, las plantaciones con Pinus pinaster y, en menor medida, sylvestris o radiata, han animado el negocio de la madera, que no ha avanzado más al mantenerse todavía mucho bosque clasificado como Patrimonio Forestal del Estado. Por lo que respecta a otros rendimientos, el monte permite obtener buenos resultados de procesos de recolección estacional, como los de castañas, setas o plantas medicinales durante el otoño. En cuanto a la agricultura en sentido estricto su importancia es muy limitada, aunque como veremos ofrece ciertas posibilidades de futuro a partir de la puesta en valor de productos de calidad. Como orientación comer cial, sólo destaca el viñedo (en estos municipios se alcanza un 17,6\% de la superficie total de este cultivo en Galicia, según datos del Censo Agrario de 1999), en espacios periféricos de las Denominaciones de Origen Ribeira Sacra, 
Figura 5. Distribución sectorial de la población activa en la montaña gallega: a) sector primario; b) Industria; c) Construcción; d) servicios.

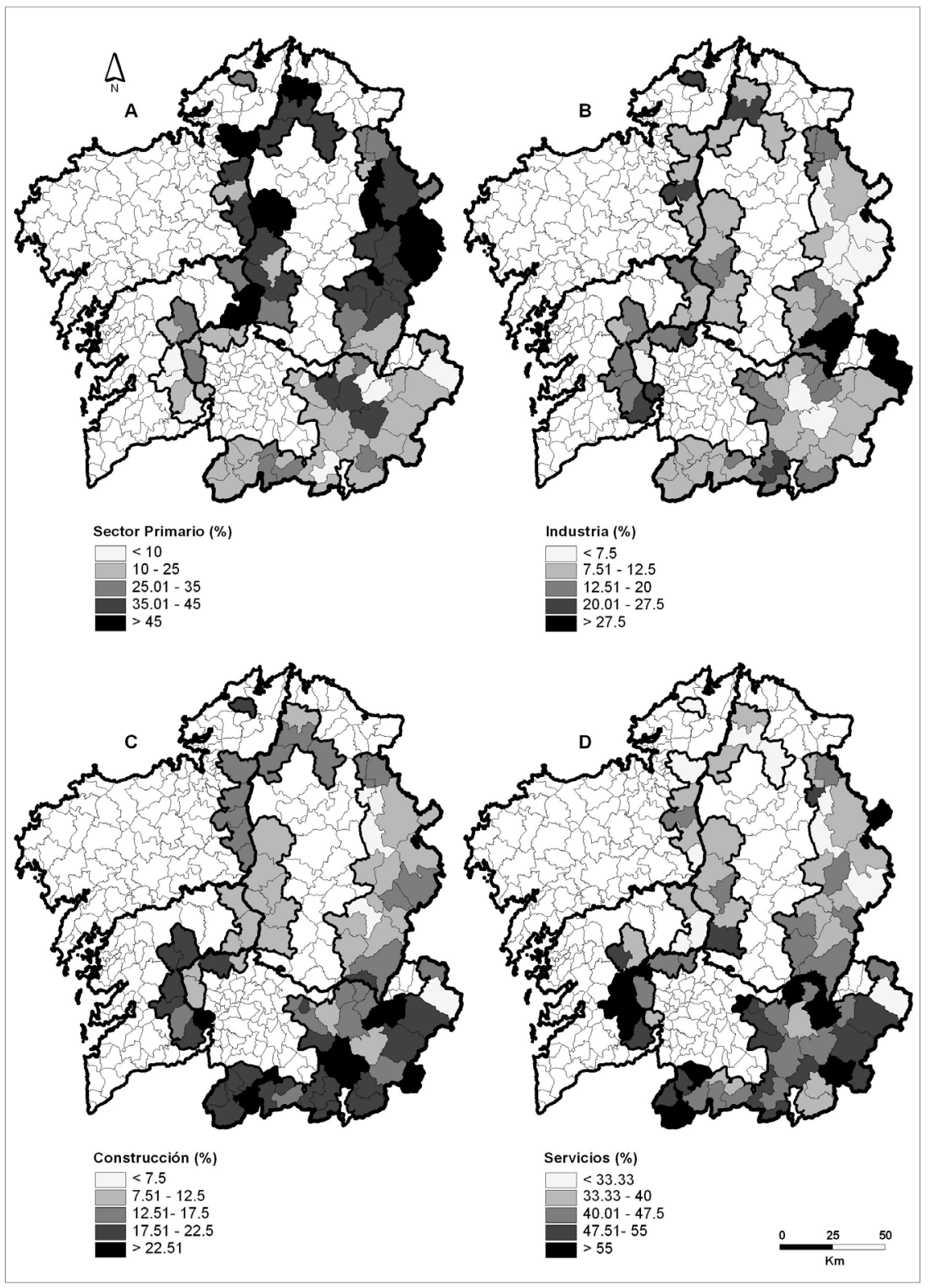

Fuente: Censo de Población y Vivienda, 2001. 
Monterrei, Valdeorras y Ribeiro. A nivel anecdótico, los últimos años han presenciado el intento de recuperación del escasísimo olivar gallego existente (23 hectáreas en las cuatro provincias), que se concentra entre los municipios de montaña de Quiroga (estribaciones del Caurel) y O Bolo (Serras de Queixa y Montes do Invernadeiro).

Como hemos comprobado, la actividad agraria que implica una menor movilización de mano de obra (por otra parte, escasa), es la que presenta una mejor implantación en la montaña gallega. De hecho, si observamos la Figura 5A se aprecia que el sector primario todavía concentra una buena parte del empleo a nivel municipal. Esto sucede de forma particular en los territorios de Lugo y A Coruña que hemos considerado, precisamente las provincias donde las dinámicas de modernización ganadera y forestal han resultado más exitosas. En Ourense y el interior de Pontevedra la mayor ocupación relativa en los sectores secundario y terciario obedece en buena medida al hundimiento de las explotaciones agrarias que no han podido adaptarse a los nuevos requerimientos de la economía de mercado (FIGURAS 5B, 5C y 5D). En los territorios ourensanos estamos, sin duda, ante sociedades perdedoras en términos de productividad y si destacan los empleados en la construcción o en el sector servicios es debido a la práctica inexistencia de una actividad agraria con proyección, más que al desarrollo de una auténtica diversificación económica. En los espacios de Pontevedra se aprecia el efecto de atracción laboral del eje Vigo-Pontevedra, y en municipios villegos como Chantada, Meira, Maceda, Póboa de Trives o A Cañiza la representatividad del terciario responde a la consolidación de mercados de trabajo característicos de cabecera comarcal.

En cuanto a la industria, la misma sólo está presente de forma minoritaria en las áreas de montaña. Destaca el empleo generado (más del 27,5\%) en Quiroga, Rubiá y Carballeda de Valdeorras, como consecuencia de la significación que alcanza la explotación de pizarra, hoy en día muy afectada por la crisis. En A Cañiza y Melón los valores obedecen a que nos hallamos ante parte de la cuenca de empleo de la ciudad de Vigo, lo mismo que Piñor de Ourense, Monterrei de Verín y Cerdido de Ferrol. Unicamente en Curtis y Muras se registran procesos locales de industrialización notables; un polígono impulsado por la SEPES para descongestionar el área A Coruña-Betanzos cerca de la A6, en el primer territorio, y el funcionamiento de una gran empresa en el segundo. Para finalizar, debemos hacer referencia a un tímido desarrollo del turismo, sólo perceptible con alguna entidad en los municipios atravesados por el Camino de Santiago (Pedrafita do Cebreiro, Triacastela, Samos y Palas de Rei), donde se localizan albergues privados, instalaciones hoteleras y hosteleras de calidad media, y casas de turismo rural. En el resto del espacio considerado, la puesta en valor comercial de ciertas áreas (Manzaneda, Trevinca, A Baixa Limia, etc.) ha permiti- 
do iniciar una incipiente orientación hacia esta actividad, con establecimientos por lo general de pocas camas que procuran ofertar los alicientes del ocio no masificado en la naturaleza.

\section{LAS POSIBILIDADES DE FUTURO PARA LAS ÁREAS DE MONTAÑA: EL EJEMPLO DE LAS BUENAS PRÁCTICAS}

A lo largo de las páginas anteriores, hemos insistido en un argumento central con matices: las áreas de montaña se encuentran en una aguda crisis, como exponentes de los espacios rurales perdedores de los recientes procesos de reconfiguración territorial. Esta crisis se manifiesta de forma impactante en términos sociodemográficos, mientras que en el plano económico la montaña gallega todavía desarrolla actividades rentables, de futuro y basadas en la puesta en valor de los recursos propios. En los siguientes párrafos, y de modo necesariamente breve, vamos a exponer qué vías pueden seguir las áreas de montaña para corregir sus elementos de decadencia, en el corto y el medio plazo. Como los modelos positivos de desarrollo endógeno no son muy frecuentes, o novedosos, plantearemos fundamentalmente una reflexión de carácter más general.

Por una parte, el principal drama que vive la montaña gallega es la magnitud de su catástrofe poblacional. Un efectivo humano notablemente envejecido y en acusado declive, que continuará dejando vacíos nuevos lugares, aldeas e incluso porciones mayoritarias de algunos municipios. Como se ha señalado, las perspectivas de futuro demográfico no son nada positivas: la población continuará descendiendo y el envejecimiento se mantendrá. No obstante, sería útil establecer un seguimiento de aquellos hogares constituidos por adultos jóvenes (20 a 40 años), originarios de los propios sectores de montaña o provenientes del exterior, que han decidido mantenerse en los espacios estudiados como opción de vida. Las disponibilidades territoriales de las áreas montañosas y la abundancia de recursos son incuestionables. Sólo se trata de animar experiencias emprendedoras, $\mathrm{u}$ opciones de vida tranquila y en contacto con la naturaleza, para a partir de las mismas formular códigos de buenas prácticas en estos espacios de mayor altitud, fuertes pendientes y una localización periférica que, sin embargo, ofrecen enormes posibilidades de futuro (KAYSER, 1990). En este sentido, la generalización de los servicios a la comunidad en unas correctas condiciones (escolarización adaptada a las bajas densidades, buen nivel de atención sanitaria y de prestación de servicios sociales, etc.), resulta fundamental para animar los procesos de repoblación de sectores especialmente difíciles.

En cuanto a las actividades económicas, el impulso a la ganadería cárnica de calidad, a la explotación sostenible del bosque y a ciertas dedicaciones agrícolas reguladas por una Denominación de Origen marcan el camino a seguir. En un mercado de alimentario cada vez más diversificado internamente (entre produc- 
tos abundantes y de bajo coste, y rendimientos amparados por indicaciones geográficas), las áreas de montaña deben orientarse a obtener menos volumen de derivados agrícolas y ganaderos, y a comercializarlos con identificadores cualitativos. A este respecto, la recuperación de razas pecuarias autóctonas, que se basan en la alimentación sobre el terreno (la vaca rubia gallega, los bovinos morenos del Noroeste, la oveja gallega, el cerdo celta, etc.), señala una vía que cuenta con un futuro envidiable. Hoy en día en Galicia existen múltiples denominaciones e indicaciones reguladas legalmente, y gran parte de las mismas extiende su área de producción por espacios de montaña. Nos estamos refiriendo a los quesos de Arzúa-Ulloa (en las estribaciones de la Dorsal), el Cebreiro (en las Serras Orientais), el San Simón da Costa (en Muras, Xermade y Abadín, en las Serras Septentrionais) o el Tetilla (por toda la montaña), a la Ternera Gallega (con diferenciaciones según los requerimientos de cada producto), a la Castaña gallega, al Pimiento de Oimbra (en el Sur de Ourense), al Lacón gallego, a la Faba de Lourenzá, a los Grelos, a las denominaciones vitícolas ya citadas (cuatro de las cinco existentes en la Comunidad Autónoma), a la Patata de Galicia, y a una lista que todavía incluye a otros rendimientos de significación menor. Como se aprecia, el marco de protección de la calidad agroalimentaria aparece creado en los sectores que estamos estudiando, ahora sólo se necesita que un mayor número de explotaciones se decida por estos productos protegidos y de calidad, para poner las bases de una nueva actividad agraria de montaña auténticamente sostenible.

De forma complementaria, diversos autores han planteado la necesidad de una explotación más racional del monte (PRADA, 1991; GUITIÁN, 2002; MIRAMONTES, 2009). En concreto, se defiende la diversificación de especies forestales y de rendimientos, que permitirían obtener beneficios suficientes a unas comunidades rurales, como hemos visto, no excesivamente numerosas. Se recomienda repoblar amplios sectores de montaña con árboles diferentes (huyendo de una marcada tendencia al monocultivo forestal), entre los que son interesantes los diversos tipos de pinos, el roble americano o los abedules, todos ellos por su potencialidad productora de madera. Todavía más importante que esta práctica, el espaciado de las plantas reduce el riesgo de incendio forestal, una amenaza real en la montaña gallega que se debe erradicar. Por último, la transformación individualizada de los distintos tipos de madera, ofrecería un modelo sostenible de rentabilización del bosque, muy alejado del actual. En la Europa septentrional existen ejemplos de gestión del monte que podrían aplicarse en Galicia, y todo el Norte de España, del mismo modo que algunas experiencias pioneras desarrolladas en municipios como Cerdido o Guitiriz también se pueden generalizar. Además, el monte no constituye sólo un ámbito de producción forestal y la potenciación de las prácticas recolectoras de sus frutos 
(castañas, setas, árnica, etc.), ofrece nuevas posibilidades a las áreas de montaña.

Una actividad que, según todos los análisis, posee una importante capacidad de crecer en la montaña es el turismo. Nos estamos refiriendo a un deseable impulso que introduzca diversificación económica en muchas áreas de atractivos paisajes y densidades muy bajas, no la sustitución de dedicaciones rurales clásicas (cultivos, cría pecuaria, etc.) ante la creación de establecimientos alojativos en el rural, como ha sucedido en múltiples ocasiones (SPARRER, 2006). El turismo debe aprovechar la existencia de un conjunto previo de trabajos agrarios para desarrollar una oferta más atractiva; en todo caso, debe rehuir convertirse en una actividad de implantación desligada de las restantes. Al mismo tiempo, y existen ejemplos muy positivos a este respecto (como la red de casas e instalaciones hoteleras que se han coordinado en Pena Trevinca), debe reforzar su especificidad impulsando una oferta complementaria de ocio en la naturaleza. De hecho, la relación entre turismo y declaración de Espacios Naturales Protegidos tiene que ser, por fuerza, directa. En la actualidad, en la montaña gallega se han conformado tres Parques Naturales (Baixa Limia-Serra do Xurés, O Invernadeiro e Serra da Enciña da Lastra), dos Monumentos Naturales (Pena Corneira y Souto de Rozabales), un Paisaje Protegido (en el valle del río Návea) y dos Reservas de la Biosfera (Ancares lucenses, y Río Eo, Oscos y Burón). A partir de esta delimitación es posible animar las visitas no masificadas de gentes que buscan el contacto con espacios poco humanizados, rutas de senderismo, el avistamiento de pájaros o la práctica de determinados deportes, entre otras posibilidades. Con el objetivo de atender esta demanda no sólo el modelo de casas rurales (bastante desarrollada) presenta las condiciones requeridas; junto a este tipo de oferta muy subvencionada hace algunos años es preciso impulsar cierta localización hotelera, de albergues privados o de campings, pues la segmentación del mercado turístico en el presente también necesita de una variedad de establecimientos y categorías alojativas para atenderla.

Para finalizar, la montaña requiere cierto impulso a su industrialización o a la creación de empresas, bajo unos determinados parámetros. En primer lugar, es poco frecuente que en las áreas estudiadas se acometan proyectos de entidad para localizar nuevos parques empresariales. Aunque en Galicia se detecta cierta saturación industrial en las áreas de Vigo, A Coruña o Santiago, es probable que la misma se corrija con la creación de polígonos en la segunda corona de estas ciudades y áreas metropolitanas, pero no en espacios mucho más periféricos (si exceptuamos el caso ya comentado de Curtis). Por lo tanto, las estrategias desarrollo industrial deben basarse en la puesta en valor de los recursos endógenos, como puede ser una mayor transformación o aprovechamiento de materiales en la extracción de pizarra (que desperdicia hasta un $98 \%$ de la roca re- 
movida), las acciones favorecedoras a la creación de pequeñas y medianas empresas del sector agroalimentario (que transformen las producciones de la montaña) o el impulso de nuevas ramas económicas (como se ha demostrado en otras regiones, las experiencias en artesanía, micromecánica o nuevas tecnologías son factibles) (BOURDESSOULE, 2002). En cualquier caso, todas estas iniciativas deberán contar con el respaldo de las administraciones públicas, obligadas a mantener criterios de discriminación positiva para las áreas de montaña. Considerando que el principal problema de los sectores elevados y periféricos es su aguda crisis poblacional, el apoyo a propuestas de apertura de empresas debe priorizar la generación de empleo, la adecuación al entorno económico y la fijación de habitantes en municipios que se están quedando vacíos. En definitiva, criterios de ordenación territorial (hasta el momento muy escasos) para facilitar el impulso a una diversificación económica con futuro.

\section{A MODO DE CONCLUSIÓN}

Durante casi dos decenios, la montaña ha sido un tema recurrente de análisis por parte de la Geografía gallega. Esto ha permitido su delimitación bajo criterios consensuados, y el estudio pormenorizado de las sierras, alineaciones, cordales y moles montañosas, según las nuevas perspectivas de la Geomorfología. A partir de esta individualización de las áreas montañosas, sistemáticamente se producen dos constataciones reiteradas, de las que este artículo tampoco ha podido escapar. Por una parte, se asume que los sectores más elevados, de fuertes pendientes y menos accesibles del país no son los escenarios preferentemente utilizados para acometer proyectos de desarrollo rural, como hubiese sido aconsejable. La montaña se conoce y reconoce en su especificidad, pero se emplea muy poco como una categoría de la ordenación del territorio (y esto sucede también en el resto de España). Por otra, todas las aproximaciones realizadas sobre el tema muestran una debacle demográfica en gran parte de los municipios de montaña. Cada nuevo artículo o contribución actualiza los valores oficiales, cada vez más dramáticos. En este sentido, sería conveniente pasar de las palabras a los hechos y convertir a las comarcas estudiadas en objetos preferentes de políticas de repoblación, o por lo menos de contención de la sangría del efectivo humano, siguiendo algunos ejemplos exitosos materializados en otras regiones europeas.

Frente a la aguda noción de crisis sociodemográfica, el repaso por las principales actividades económicas de la montaña todavía muestra su vitalidad en ciertos sectores. Aunque una parte de los territorios estudiados (en especial, los situados en la provincia de Ourense), expresan en la actualidad una marcada atonía productiva, en otras áreas (por ejemplo, en la Dorsal o en las Serras Orientais) sorprende la capacidad de adaptación de la ganadería de montaña a los nuevos tiempos, así como las estrategias de rentabilización forestal o ciertas 
iniciativas estrictamente agrícolas. También hemos comprobado la movilidad de las personas que viven o trabajan en la montaña. Muchos jóvenes optan por continuar residiendo en sus aldeas de origen, y se desplazan a una ciudad o una cabecera comarcal próxima para cumplir su jornada laboral. De modo paralelo, numerosos empresarios residentes en pequeñas ciudades o villas mantienen explotaciones mineras, del bosque o pecuarias en sectores relativamente alejados de montaña. Los espacios estudiados ofrecen un dinamismo mayor que el que cabría esperar. En todo caso, la ausencia de auténticas políticas favorecedoras de la diversificación productiva (más allá de las declaraciones en este sentido de los organismos que gestionaron, y despilfarraron, fondos europeos) y de actuaciones guiadas por criterios de ordenación del territorio, todavía limita un auténtico desarrollo turístico, empresarial y agroalimentario en estos sectores, que deberían ser protegidos en beneficio de toda la comunidad.

\section{BIBLIOGRAFÍA}

ALDREY VÁZQUEZ, J.A. (2009): «Implicaciones socioterritoriales de la despoblación en la montaña astur-galaico-leonesa», en (LÓPEZ TRIGAL, L. et al., Coords.): Envejecimiento, despoblación y territorio. León, Universidad de León, 547-566.

BEIRAS, X.M. (1972): O atraso económico de Galicia. Galaxia, Vigo.

Bordessoule, E. (Coord.) (2002): Questions de Géographie. Les Montagnes. du Temps, Nantes

BOUHIER, A. (1979): La Galice. Essai d'interpretation d'un vieux complexe agraire. II Vols. Imp. Yonnaise, La Roche-Sur-Yon.

CABERO, V.; MAJORAL, R y RODRíGUEZ MARTÍNEZ, F. (1992): «Las áreas de montaña y su problemática», en (REAL SOCIEDAD GEOGRÁFICA Y ASOCIACIÓN DE GEÓGRAFOS ESPAÑOLES), La Geografia en España (1970-1990). Aportación Española al XXVII ${ }^{\circ}$ Congreso de la Unión Geográfica Internacional, 247-263. Fundación BBV, Madrid.

CEE, DireCtiva 268/75, de 28 de Abril de 1975, sobre agricultura de montaña y determinadas zonas desfavorecidas en el territorio comunitario.

CIMA (Colectivo de Investigadores sobre las Montañas Españolas) (2005): Las montañas españolas conceptos y vocabulario. Entinema, Madrid.

COLINO, X. y PÉREZ TouriÑo, E. (1983): Economía campesiña e capital. A evolución da agricultura galega (1960-1980). Galaxia, Vigo.

ERIA (2008): Monográfico $\mathrm{n}^{\circ} 75$, dedicado a las áreas de montaña. Universidad de Oviedo. Oviedo.

GARCÍA FERNÁNDEZ, J. (1975): Organización del espacio y economía rural en la España Atlántica. Siglo XXI, Madrid.

Grupo ADUAR (2000): Diccionario de geografia urbana, urbanismo y ordenación del territorio. Ariel, Barcelona.

GuITIÁN, L. (2002): «La destrucción histórica del bosque en Galicia» en (L. GUITIÁN y A. PÉrez Alberti, Eds.), Historia ecológica de Galicia. Revista Sémata, no 13, 105-167. Universidade de Santiago de Compostela, Santiago de Compostela.

KAYSER, B. (1990): La Renaissance Rurale. Sociologie des campagnes du monde occidental. Armand Colin, Paris. 
LOIS GONZÁleZ, R.C. y RodRÍGUEZ GONZÁLEZ, R. (2001): «Cambios recentes no espacio rural: a Galicia interior», en (SAN RomÁn RodRíGUEZ, J. Mª ., Coord.), IX Semana de Historia. Instituto de Estudios Valdeorreses. Cuaderno Monográfico, $\mathrm{n}^{\mathrm{o}} 35$. 29-53. IEV, Madrid.

Lois GonzÁlez, R.C., SAn Román Rodríguez, J. Mª y Aldrey VÁzQuez, J.A. (2008): «El impacto de la actividad minera en la población de los espacios de montaña: La pizarra y el carbón en las montañas galaico-leonesas», Ería, n 75, 99-112.

LÓPEZ SiLVESTRE, F. y SOBRINO MANZANARES, Mª.L. (EDS.) (2006): Nuevas visiones del paisaje: la vertiente atlántica. Centro Galego de Arte Contemporáneo, Xunta de Galicia, Santiago de Compostela.

MARTínEZ DE PISÓN, E. (1980): «Los conceptos y los paisajes de montaña», en Superviviencia de la Montaña, 21-34. MAPA, Madrid.

MirAmontes CARBALlADA, A. (2009): La industria de la madera en Galicia. La significación del subsector del mueble. Tesis Doctoral (Inédita). Departamento de Xeografía. Universidade de Santiago. Santiago de Compostela.

MuÑoz JimÉnEZ, J. y SANZ HeRrÁIZ, C. (1995): Las montañas, Guía Física de España, $\mathrm{n}^{\circ}$ 5. Alianza Editorial, Madrid.

Prada Blanco, A. (1991): Monte e industria. O circuito da madeira en Galicia. Fundación Caixa Galicia, A Coruña.

RODRÍGUEZ GONZÁlEZ, R. (Dir.) (2004): Os concellos galegos para o século XXI. Análise dunha reestructuración do territorio e do goberno local. Universidade de Santiago y FEGAMP. Santiago de Compostela.

SAN RoMÁn RodríGUEZ, J. Ma (2000): Valdeorras. La industria de la pizarra y las transformaciones espaciales. Municipios de O Barco, Carballeda y Rubiá (19501998). Cuaderno Monográfico $\mathrm{n}^{\circ} 32$. IEV, Madrid.

SAN ROMÁN RODRÍGUEZ, J.Ma . (2005): Las transformaciones espaciales recientes en las áreas montañosas colindantes entre Ourense y Castilla y León: un estudio de geografía regional. Tesis Doctoral (Inédita). Departamento de Xeografía. Universidade de Santiago. Santiago de Compostela.

SPARRER, M. (2006): O turismo en espazo rural como unha estrategia de desenvolvemento. Unha comparación a nivel europeo. Xunta de Galicia, Santiago de Compostela.

Torres LunA, M.P., Lois GonzÁlez, R.C. y PÉrez Alberti, A. (1993): A Montaña Galega. O Home e o medio. Universidade de Santiago de Compostela, Biblioteca de Divulgación, Santiago de Compostela.

TORRES LunA, M.P. y LOIS GonZÁLEZ, R.C. (2009): O Territorio, en (GARCÍA PAZOS, F., Coord. Ed.) A parroquia en Galicia: pasado, presente e futuro, 19-54. Xunta de Galicia, Santiago de Compostela. 